\title{
Ananalysis on Runtime Associated QOS-Based Proficient Web Services Discovery Optimization
}

\author{
A. Amirthasaravanan ${ }^{1 *}$, Paul Rodrigues ${ }^{2}$, R. Sudhesh ${ }^{3}$. \\ ${ }^{I}$ Department of Information Technology, University College of Engineering, Villupuram, Tamilnadu, India; \\ ${ }^{2}$ Department of Computer Science and Engineering, DMI Engineering College, Chennai, Tamilnadu, India; \\ ${ }^{3}$ Department of Mathematics, University College of Engineering Villupuram, Tamilnadu,India.
}

\begin{abstract}
In today's web world, Service-oriented architectures represent the main standard for IT infrastructures. Certainly, with the initiation of service oriented architecture, Web services have gained incredible growth. Web service discovery has become increasingly more significant as the existing use of web service. Discovering most appropriate web service from vast collection of web services is very decisive for successful execution of applications. In automation of web service discovery, there is always a need to deem Quality of Service (QoS) attributes during matching. A study of literature concerning the evolution of different web service discovery optimization methods with unique prominence to quality motivated service discovery have been carried out in this work. This paper depicts Bio-inspired algorithms optimizing the discovery process for semantic web services. Bioinspired algorithm is a metaheuristics method that mimics the nature in order to unravel optimization difficulty and evaluates the analysis of some popular bio-inspired optimization algorithm systematically. This paper also focused on the principle of each algorithm and their application with respect to run time oriented QoSattributes and from result the best suitable bio-inspired optimization algorithm is been deployed.
\end{abstract}

Key words: Web Services, Service-Oriented Architectures, Discovery, Quality of Service, Bio-Inspired Algorithm, Metaheuristics, Optimization.

\footnotetext{
*Author for correspondence: aasaravanan777@gmail.com
} 


\section{INTRODUCTION}

Web services are open standard, autonomous software services that can be accessed on network using effortless protocols. Web services flourish; apprehension includes the overall demands on network bandwidth for any particular service, the influence on performance as demands for that service growth. It has an interface expressed in a machine- process able presentation like WSDL (WSDL is used for describing the services available). ${ }^{[2]}$ Other systems cooperate with the web service in a approach prescribed by its description using SOAP (SOAP is used to transfer the data) messages, typically communicated using HTTP with an XML (XML is used to tag the data) serialization in union with other web-oriented standards. UDDI is used for listing out the services available. Web Services can be executed and used in nearly all Programming Languages.

The Web services architecture has three roles which interact with each other and produces web services :

- Service Provider

- Service Registry

- Service Requestor

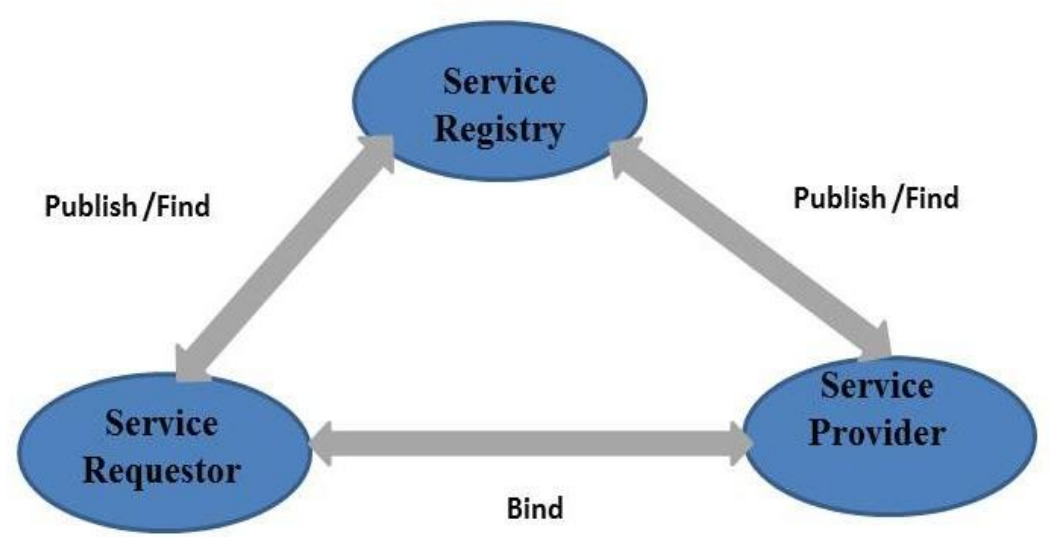

Figure.1.Web Services Architecture

- Service Provider: The main job of service provider is it depicts a service description to the web service and then publishes it to a service registry or service requestor.

- Service Requestor: Uses a discover operation to retrieve the service description from the service registry or locally and binds the service description to the service provider and work together with the Web service completion.

- Service Registry: The registry offers a central and vital place where developers can publish new services or find existing services.

${ }^{[2]}$ Publish operation where, a service description needs to be published and the Published service requestor can locate it. Find-In this operation, the service requestor retrieves service descriptions discover directly or queries the service registry for the type of service. Binding is invoking operation of services based on the service description. Web Services have gained popularity for many reasons 
such as interoperability, maintenance, and reuse. The most commonly accepted and triumphant type of service is the XML Web service.

The web services are designed for interoperability characteristics. It recognizes the universal elements of the universal web services network that are mandatory in order to guarantee interoperability among Web services. The web services architecture state both the negligible characteristics that are aware to every web services and a number of characteristics that are desired by many web services.

\section{QUALITY OF SERVICE}

Web services have the latent to be composed to cross organizational workflows. Since growing number of web services provide identical functionalities, more significance is being placed on how to discover the service that finest fits the consumer's or user requirements. With the rapidly rising number of web services with akin or identical functionality, the non-functional properties of a web service will turn into more and more considerable day by day. Apart from costs for using web services, ${ }^{[10]}$ the Quality of Service (QoS) attributes like Response time, Accuracy, Availability and Throughput are included as non-functional attributes. In order to discover services that best meet their QoS provisions, the service consumers or users and discovery agents need to distinguish between the QoS information for the services and the trustworthiness of this information.

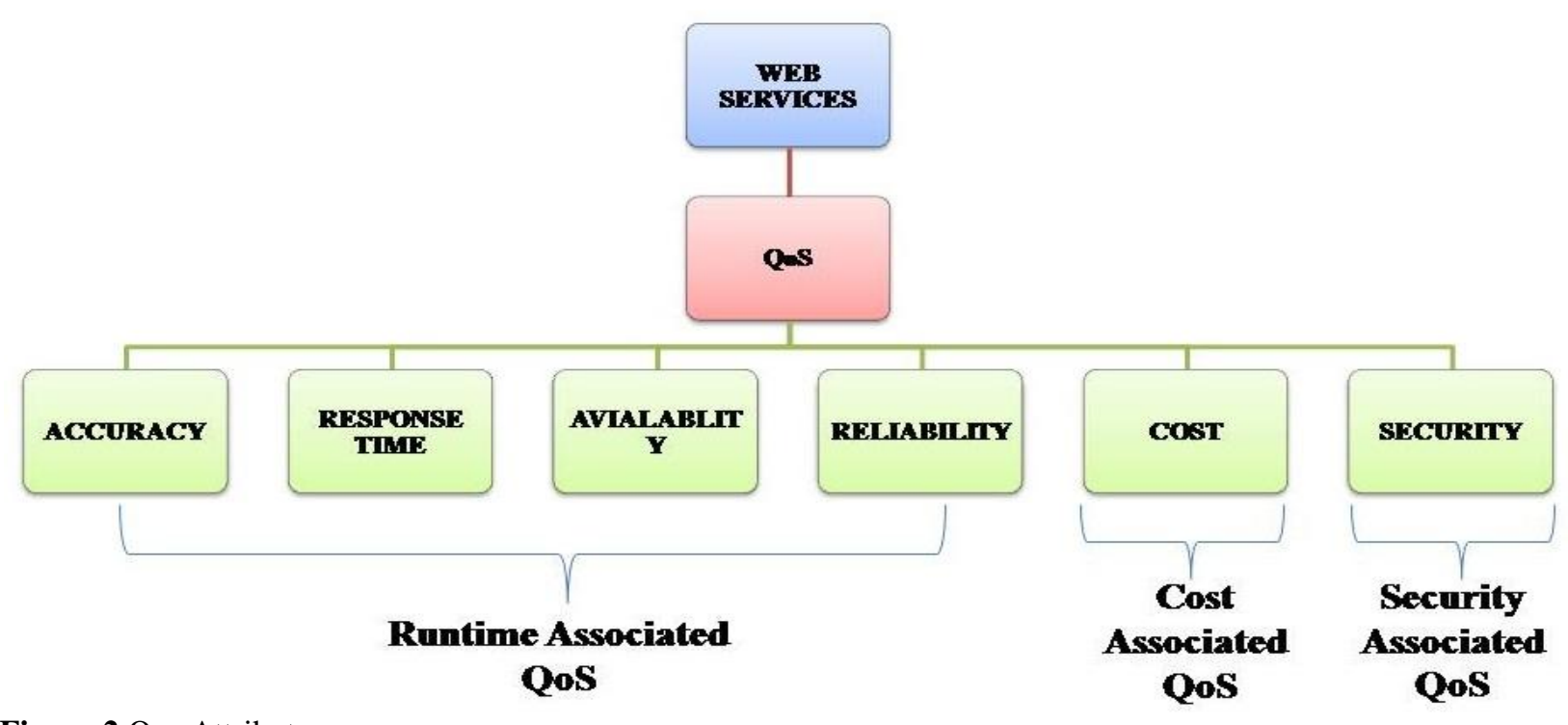

Figure.2.Qos Attributes

The Quality of Services can be characterized according to different dimensions called QoS attributes or QoS classes. QoS attributes are like availability, reliability, and reputation. This work has chosen two following QoS attributes for doing research; however it can flexibly take any other attribute into account.

\section{QoS ATTRIBUTES}

Accuracy: ${ }^{[10]}$ Accuracy is a measure of appropriateness or correctness delivered by a web service. The number of errors produced by a Web service, fatal errors and the frequency of the condition determine the amount of accuracy for a web service. Accuracy is the level to which web services give correct results for the received 
requests. Accuracy of the web service is premeditated by the standard deviation of the reliability. As the average value of the standard deviation is equivalent to zero, the measurement is believed to be accurate. If the standard deviation average value is very high, then the measurement is supposed to be not accurate.

Response Time: ${ }^{[10]}$ The delay time in milliseconds between sending a request and receiving the results. This duration consists of the processing time of the service and data transmission time. The response time is the time when the Server responds after processing the request. Apparently, the throughput and the response time rely on the task that the web server is undergoing at that time. Both throughput and latency can be calculated by keeping track of the timestamps at the request time and response times. The latency of any request processing is the difference between the timestamps equivalent to the request time and response time, and the throughput is given by the sum of requests divided by the elapsed time between the request time and the response time.

Availability: ${ }^{[10]}$ Availability is the probability that the web service is up and in a gladly functioning state. High availability assure that there is the least amount of system failures or server failures even during the peak times when there is hefty traffic to and from the server and that the given service is available relentlessly at all times. Let us say the "down time" is when a system is not available. As the system is either available or unavailable, the remaining time after subtracting the down time can be termed as the "up time" that means the system is available. A checking upon down time is easier than up time .We can consider to calculate down time to measure the availability. Keeping a tab on the events failed during an operation can possibly give the down time.

Reliability: ${ }^{[10]}$ Reliability is measurement of quality of a web service in terms of performs, given an explicit amount of time and the present network conditions, while sustaining the service quality. It is also resolute by the number of failures per day and the means of delivery. The tally on failures is based on the number of duplicate deliveries, flawed message deliveries, dropped deliveries and out-oforder deliveries. An event may either be successful or failed, and there is no middle ground in that concern. Thus, sum of events will be the number of failures added to the sum of successful events.

\section{OPTIMIZATIONALGORITHMS}

Optimization is an endeavor of attaining the optimal solution of problem under given conditions. The decisive task of optimization is to reduce wasted time or maximize desired benefit of a given system. ${ }^{[7]}{ }^{[9]}$ All the systems that are to be optimized have an objective function and some decision variables that influence the function optimization methods can be defined as a process of accomplishing optimal solutions that assure a given objective function.

Optimization algorithms are generally divided into two groups as deterministic and stochastic algorithms. Deterministic algorithms do not include any operators that cause randomness. This type of algorithms produces the similar result as long as their original conditions remain constant. On the other side, due to their random nature, stochastic algorithms are inclined to create different solutions even when their original conditions remain constant at each run. 


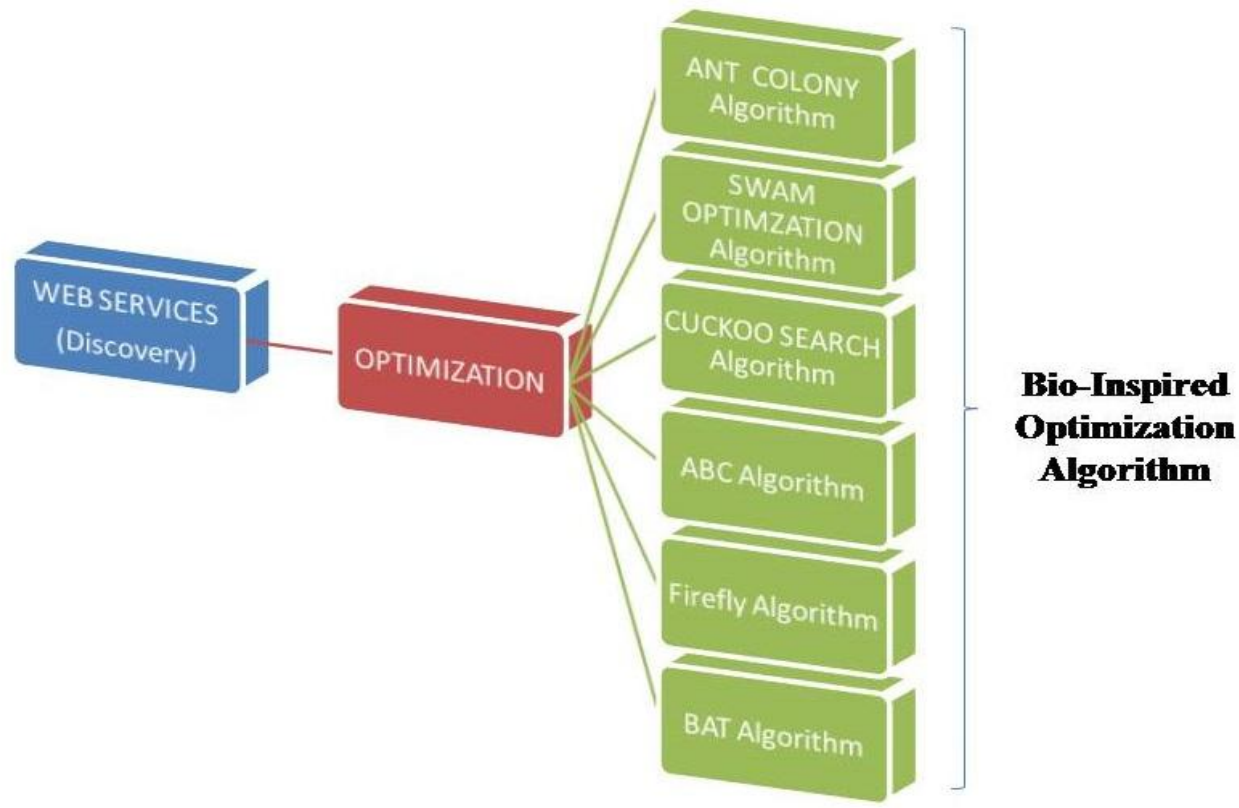

Figure.3.Bio Inspired Optimization Algorithm

Stochastic algorithms are ideal for such functions as they can get away from local minima easily in spite of their slow convergence speed. Stochastic algorithms are categorized into two groups such as heuristic and metaheuristic algorithms. Heuristic algorithms produce high quality results by trial and error methods in a suitable computational time. The studies in the literature tend to refer to all novel stochastic algorithms as metaheuristic. Metaheuristic utilizes localization and random search .Heuristic algorithms are normally inspired by the nature; hence these algorithms are also called nature or bio inspired algorithms. Flexible and straightforwardly applicable structure of these algorithms has made them very well accepted in recent years.

Bio inspired algorithms plays a vital role in solving complex real world problem. Amid them the most triumphant algorithms are Swarm Intelligence algorithms and Evolutionary algorithms. The perception of copying the nature into technology is demonstrated to be successful in optimization as described in many literatures.

These techniques are being studied and worked in practice at an increasing rate. Their robustness capability of providing from single to multiple solutions of problem and appropriateness of implementation in distributed computing environment makes them a powerful problem resolving tool. Furthermore, bioinspired algorithms are also capable to overcome many shortcomings of habitual algorithm applications. This paper presents an analysis of some bio inspired optimization methods that are being implemented in many distributed computing environment research areas.

\section{BIO-INSPIRED ALGORITHMS}

\section{ANT COLONY ALGORITHM}

Ant Colony ${ }^{[3]}$ Optimization algorithm is a metaheuristic optimization algorithm and probabilistic technique for resolving computational problems. It was first initiated by Marco Dorigo in 1992. Ants stroll randomly to finding food and return to their colony while laying down pheromone traces. If other ants find such a path, they are liable not to continue travelling at random, but to follow the traces, returning and emphasize it if they finally find food. When one ant finds a shorter 
path from the colony to a food source, other ants are more liable to follow that path, and optimistic feedback ultimately leads to all the ants' following a single path. The design of the ant colony algorithm is to imitate this behavior with "simulated ants" walking around the graph in lieu of the problem to solve.

\section{PARTICLE SWARM ALGORITHM}

Particle Swarm optimization (PSO) ${ }^{[13]}$ is a computational acumen oriented, stochastic, population-oriented global optimization technique proposed in 1995by Kennedy and Eberhart. It is inspired by the community behavior of bird flocking in search for pray. PSO has been broadly applied to many engineering optimization fields due to its exceptional searching mechanism, computational efficiency, simple concept and easy implementation. In PSO, the term - particles refers to population members who are volume-less, mass-less and are subject to velocities and accelerations for a better form of behavior. Each particle in the swarm symbolize a solution in a high-dimensional space by four vectors, its present position, best position originated so far, the best position originated by its neighborhood so far and its velocity and fiddle with its position in the search space based on the best position reached by its neighborhood during the search process and on the best position reached by itself.

\section{CUCKOO SEARCH ALGORITHM}

Cuckoo Search ${ }^{[1]}[16]$ is an optimization algorithm developed in year 2009 by Yang and Deb. Cuckoo Search, is inspired by the life style of a bird family. Cuckoo birds have an antagonistic mock in which females cuckoo seize and lay their fertilized eggs in other birds' nests. If the host bird understands that the egg does not belong to them, it either discards or chucks away from its nest and put up a new one elsewhere. This algorithm works on three assumptions:

Assumption 1: Each cuckoo lay an egg, which symbolize a set of solution coordinates, at a time and puts it in a random nest.

Assumption 2: A part of the nests comprising the best eggs, or solutions, will take over to the successive generation.

Assumption 3: The number of nests is untouched and there is a probability that a host bird can discover an alien egg. If this arises, the host bird can either discard the nest or the egg and fact that this result in building a new nest in a new place.

An vital advantage of this algorithm is its effortlessness implementation and when comparing with other metaheuristic algorithms like particle swarm optimization and harmony search, there is fundamentally only a single parameter in CS. Therefore, it is very easy to implement.

\section{ARTIFICIAL BEE COLONY ALGORITHM}

AB Colony (ABC) ${ }^{[6]}$ Optimization algorithm is a probabilistic method for solving computational problems which can be condensed to finding good paths all the way through graphs. Artificial Bee Colony is a principal algorithm simulating the intelligent foraging behavior of a honeybee group, which was proposed by Karaboga and Basturk. In ABC algorithm, artificial bees' colony contains three groups of bees: onlookers bees, employed bees, and scouts bees. A bee waiting on the dance area for making a decision on food source is called onlooker and one traveling to the food source visited by it before is called as employed bee. The other type of bee is scout bee travels randomly for discovering new food sources. The position of a food source symbolizes a feasible solution to the optimization problem and the nectar amount of a food source corresponds to the quality of the solution. 
A group of virtual bees is generated and started to move randomly in twodimensional search space. Bees cooperate with each other when they find some target nectar and the solution of the problem is attained from the intensity of these bee interactions. The confined search performance of $\mathrm{ABC}$ algorithm depends on neighborhood search and greedy selection mechanisms performed by onlooker bees and employed. The global search performance of the algorithm depends on random search process performed by scouts and neighbor solution production mechanism performed by employed bees and onlooker bees.

\section{FIREFLY ALGORTHM}

Firefly Algorithm (FA) ${ }^{[18]}$ is stimulated by the flashing behavior of fireflies and it is a metaheuristic algorithm. The firefly relies on fireflies which communicate with one another to solve optimization problems. The behavior of artificial fireflies is formed based on the behavior of fireflies in nature, which look for a mating partner by emanating a flashing light. We present how we correlated the perception of the firefly (Bio inspired) metaheuristic algorithm to the problem of web service composition.

It was proposed by Xin-She Yang assuming:

- All fireflies are unisexual, due to which one firefly will be attracted to all other fireflies.

- Attractiveness is qualified to their brightness, and for any two fireflies, the less bright firefly will be attracted by the brighter one; but, the brightness can decrease as their distance increases.

- If there are no fireflies brighter than a given firefly, it will travel randomly. The brightness should be related with the objective function.

\section{BAT ALGORITHM}

Bat Algorithm (BA) ${ }^{[1][17][19]}$ it is a metaheuristic optimization algorithm based on echolocation of microbats with changeable pulse rates of emission and loudness. And it was developed in the year 2010 by Xin-She Yang .

The microbats use echolocation widely as sensor to detect obstructions. Theses bats produce sound pulse and sense for echo as it coil back from surroundings with sum time delay. All bats use echolocation to sense obstructions and distance. The bats know the difference between hindrance and prey. A bat flies arbitrarily with velocity at location with a fixed frequency, unreliable wavelength and loudness in search for prey. The frequency or wavelength of produced pulse can be adjusted robotically as well as the rate of pulse emission which is depending on nearness of the objective. The loudness can be contradictory from large (positive) to minimum value. In simple terms the Algorithm can be represent as each virtual bat flies arbitrarily with a velocity at position with a varying frequency and loudness. By way of it examines and finds its prey, it fluctuates regularity, loudness and pulse emission rate. Exploration is strengthened by a local arbitrary walk. Selections of the best keep on until stop criteria are met.

\section{ASSUMPTIONS FOR EMPIRICAL EVALUATION}

The QoS is a nonfunctional attribute which is indirectly propositional to the request (that is the user requesting for a services).In this paper the performance of every described metaheuristic algorithm is evaluated and compared in order to observe the Response time and accuracy criteria towards comprehensive optimum . In order to perform the same we consider 2 major QoS run time associative attributes like Response time and Accuracy. 
In order to evaluate the Algorithm, we consider 3 categories of assumptions for the user request:

1. Well Define (WD): The user request is well defined. Then the scope of the user request is unambiguous and the formation of keywords is semantic in nature.

2. Moderate (M): The user request is not well defined but it is averagely distinct. Then the scope of the user request is temperate ambiguous and formation of keywords are not clear as semantic in nature. It restrains redundant keywords.

3. Blind (B): The user is not sure of about his request. Then the scope of the user request is hazy and there is no formation of correct keywords. It holds improbability keywords.

Table.1.Catigorizes of User Request

\begin{tabular}{|l|l|l|l|}
\hline USER REQUEST & $\begin{array}{l}\text { USER REQUEST } \\
\text { VALUE } \\
\text { (ASSUMPTION) }\end{array}$ & RESPONSE TIME & ACCURACY \\
\hline $\begin{array}{l}\text { Well defined Search } \\
\text { Request(WD) }\end{array}$ & 1 & Less & High \\
\hline $\begin{array}{l}\text { Moderate Search } \\
\text { Request(M) Search }\end{array}$ & 0.5 & $\begin{array}{l}\text { Moderate comparatively } \\
\text { More then (WD) }\end{array}$ & Fair \\
\hline $\begin{array}{l}\text { Blind to } 0 \\
\text { Request(B) } \quad \text { More }\end{array}$ & Comparatively Less \\
\hline
\end{tabular}

The Response time is the time when the web services server replies after processing the request of the user. For evaluation purpose we propose response time as:

UserRequest $\propto \frac{1}{\text { Response Time }} \ldots 1$

As mentioned above there are 3 types of user request, we consider three cases for Response time correlated to user request:

Case 1: If the request is Well Defined by the user to the service requestor, the total time to respond to the query (request) will be minimal. (The searching time is relatively becomes less)

Case 2: If request is Moderate by the user to the service requestor then the total time to respond to the query (request) will be temperate. (The searching time is plausibly become lofty as compared to well defined)

Case 3: In this case if the user request is blind or improper search (the query content is not clear). The total time to respond to the query (request) will be significantly increased. (The searching time is literally becomes high) or it will not provide any services. Accuracy is defined-- as the level to which web services give the correct results for the user request.

UserRequest $\propto$ Accuracy....2

Similar to the Response time, we consider three cases for Accuracy correlated to user request:

Case 1:If the request is Well Defined by the user to the service requestorand the resultant is unambiguous, then Well Defined user request will have high Accuracy.

Case 2: If the request is framed moderately i.e. the request is temperate ambiguous and formation of keywords are not clear as semantic in nature, it restrains redundant keywords. Then the resultant services generated will also be with reticent Accuracy. 
Case 3: If the user is not sure(Blind) about his request, then the request is consider as improper or blind search and due to which there is a holdup in accuracy or zero accuracy.

From the equations 1, 2 we formulate user request as:

User Request $\propto \frac{\text { Accuracy }}{\text { Response Time }} . .3$

By formulating the equations 1 and 2 we infer that the User Request is directly propositional to accuracy and inversely propositional to Response time. A good web services discovery Algorithm should take less Response Time to process the request and high accuracy towards resultant of the request. This intern lead to effective QoS based optimization of web services discovery. We have presented below a comparative analysis of Bio inspired algorithms in the form of a table and we also consider two most important Runtime associated QoS attributes like Response time and Accuracy, since the Reliability and availability are closely related to Accuracy and Response time.

More over the Accuracy and the Response time are the current important issue of today web service discovery. Since we do not have any currently recognized standard platform and dataset which are used to measure web services discovery, this paper obtain web services selected by webxml, Google search and XMethod website as test data for this research. This dataset has been used since it contains varied topics from different fields like art, geography and sports etc.

Table.2.Comparision Table for Bio-Inspired Algorithm

\begin{tabular}{|c|c|c|c|c|c|}
\hline METHOD & $\begin{array}{c}\text { PROPOSED } \\
\text { BY }\end{array}$ & YEAR & \multicolumn{2}{|c|}{$\begin{array}{c}\text { RESPONSE TIME } \\
\text { m.s }\end{array}$} & $\underset{\%}{\operatorname{ACCURACY}}$ \\
\hline \multirow{3}{*}{ PARTICLE SWARM } & \multirow{3}{*}{$\begin{array}{l}\text { Kennedy and } \\
\text { Eberhart }\end{array}$} & \multirow{3}{*}{1995} & WD & $0.34 \mathrm{~ms}$ & \multirow{3}{*}{43.7772} \\
\hline & & & $\mathrm{M}$ & $0.47 \mathrm{~ms}$ & \\
\hline & & & B & $0.62 \mathrm{~ms}$ & \\
\hline \multirow{3}{*}{ ANT COLONY } & \multirow{3}{*}{$\underline{\text { Marco Dorigo }}$} & \multirow{3}{*}{1992} & WD & $0.67 \mathrm{~ms}$ & \multirow{3}{*}{39.4575} \\
\hline & & & $\mathrm{M}$ & $0.55 \mathrm{~ms}$ & \\
\hline & & & $\mathrm{B}$ & $0.69 \mathrm{~ms}$ & \\
\hline \multirow{3}{*}{ FIREFLY ALGORTHM } & \multirow{3}{*}{ Xin-She Yang } & \multirow{3}{*}{2007} & WD & $0.44 \mathrm{~ms}$ & \multirow{3}{*}{60.0124} \\
\hline & & & $\mathrm{M}$ & $0.47 \mathrm{~ms}$ & \\
\hline & & & B & $0.51 \mathrm{~ms}$ & \\
\hline \multirow{3}{*}{$\begin{array}{l}\text { ARTIFICIAL BEE } \\
\text { COLONY }\end{array}$} & \multirow{3}{*}{$\begin{array}{l}\text { Karaboga and } \\
\text { Basturk }\end{array}$} & \multirow{3}{*}{2005} & WD & $0.36 \mathrm{~ms}$ & \multirow{3}{*}{49.3224} \\
\hline & & & M & $0.43 \mathrm{~ms}$ & \\
\hline & & & B & $0.57 \mathrm{~ms}$ & \\
\hline \multirow{3}{*}{ CUCKOO SEARCH } & \multirow{3}{*}{ Yang and Deb } & \multirow{3}{*}{2009} & WD & $0.21 \mathrm{~ms}$ & \multirow{3}{*}{92.3319} \\
\hline & & & $\mathrm{M}$ & $0.32 \mathrm{~ms}$ & \\
\hline & & & $\mathrm{B}$ & $0.44 \mathrm{~ms}$ & \\
\hline \multirow{3}{*}{ BAT ALGORITHM } & \multirow{3}{*}{ Xin-She Yang } & \multirow{3}{*}{2010} & WD & $0.31 \mathrm{~ms}$ & \multirow{3}{*}{76.0533} \\
\hline & & & $\mathrm{M}$ & $0.44 \mathrm{~ms}$ & \\
\hline & & & B & $0.50 \mathrm{~ms}$ & \\
\hline
\end{tabular}

The test has been made based on 75 request (each 25 categories of request for WD,M,B) by the user.The scope of user request are categorized as Well defined and moderate and blind request .

For example a request like "Gold mine in south Africa" is categorized (assumed) as well defined Request." Gold mine" is categorized (assumed) as moderate Request. 
And "mines in the world"or "mines" is categorized (assumed) as blind Request. Response time is definite maximum (or average or min) time necessary to complete a service request.

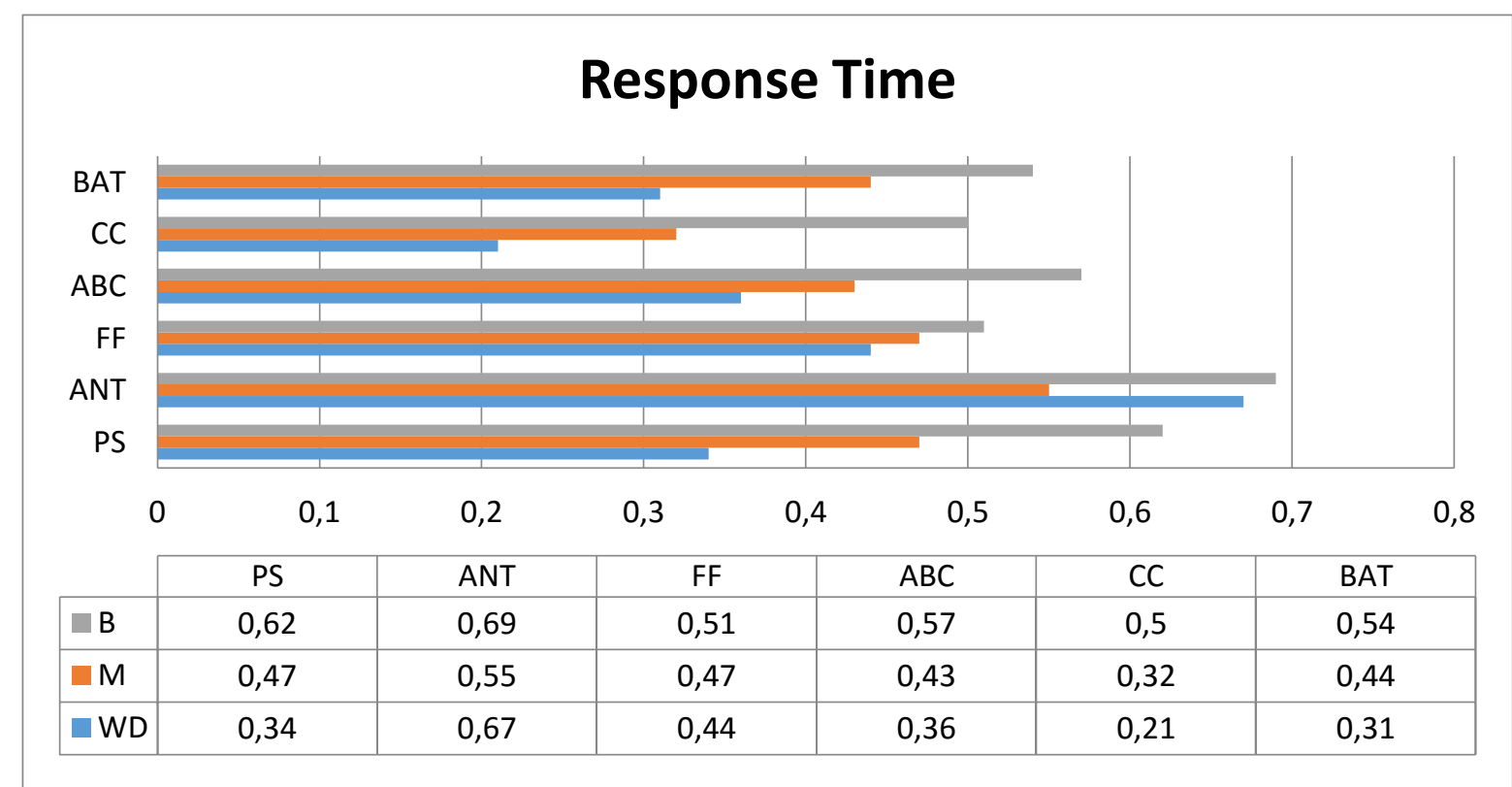

Figure.4. Evaluation result for response time of user request classifications for different Bio Inspired Algorithm

The graphs above show the variation of response time for various types of user request. In figure.4. Graph shows the response time (milliseconds) of user request classifications for different bio-inspired algorithms, in X-axis and list of optimization algorithms in Y-axis. Response time varies with time required to respond to a user request. (By each 25 categories of request for WD,M,B)

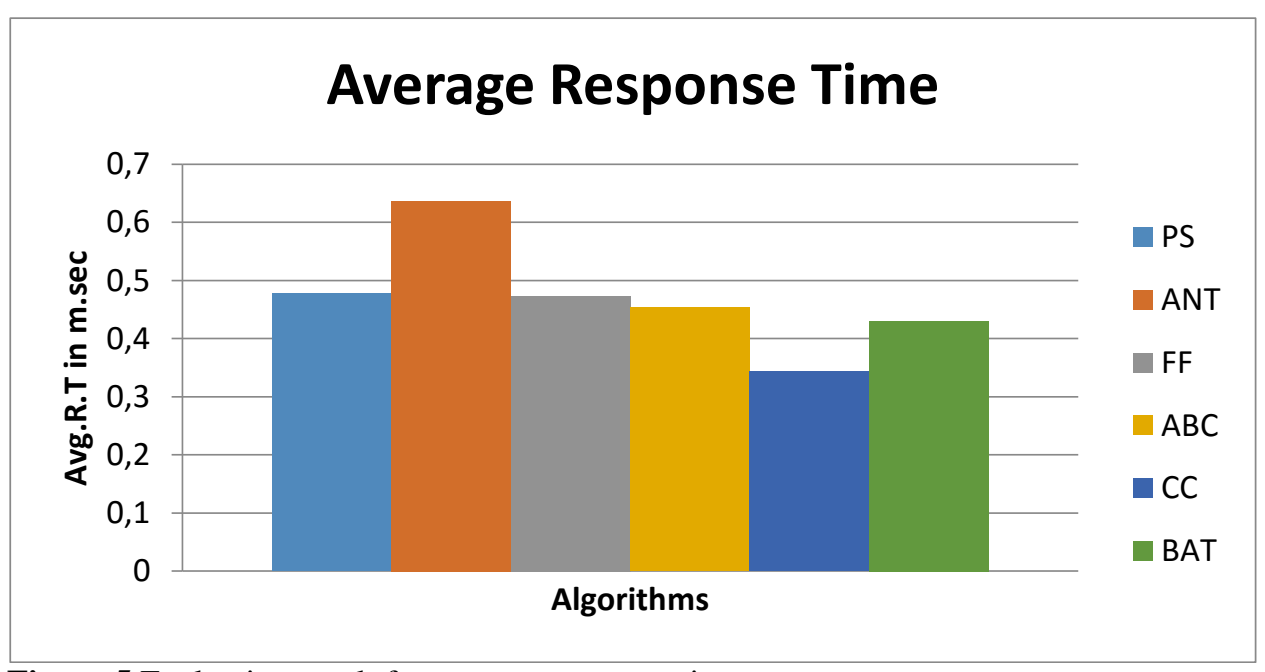

Figure.5.Evaluation result for average response time

The graph above shows the variation of average response time for various types of user request. In the figure.5 graph shows the response time (milliseconds) of user request classifications for different bio-inspired algorithms, in Y-axis and list of optimization Algorithms in X-axis. Average Response time is calculated by taking average mean of all user request classifications. From the analysis we infer that 
cuckoo search algorithm has less response time compare to other Bio inspired algorithm.

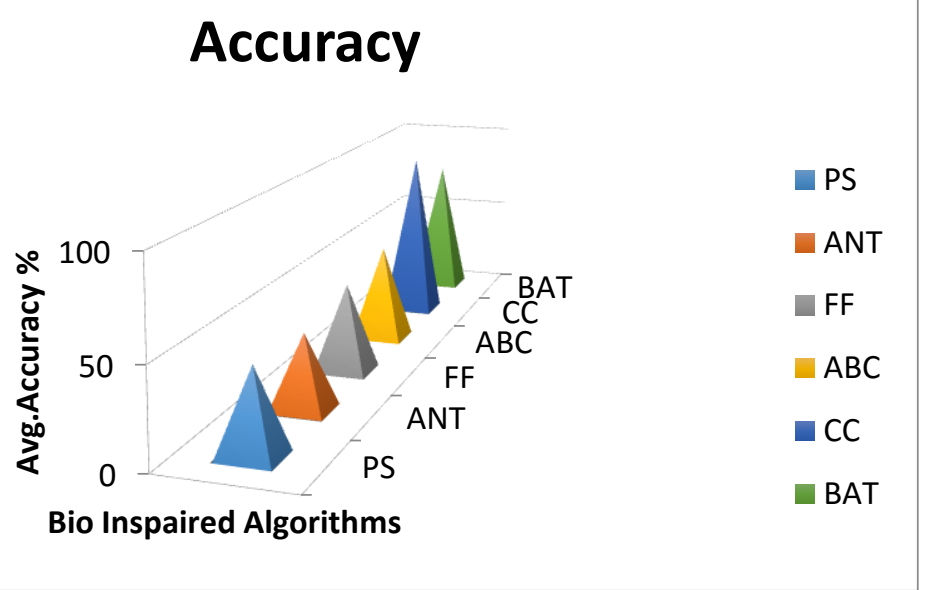

Figure.6. Evaluation result for Average Accuracy of Bio Inspired Algorithm

The graph (Figure.6) above shows the level of Accuracy of different Bio Inspired Algorithm. The accuracy level is given in Y-axis and list of Bio inspired optimization Algorithms in X-axis. (By each 25 categories of request for WD, M, B). Accuracy is where Web services give correct results for the received user requests.

\section{RESULTS AND CONCLUSION}

From our above analysis we infer that the best algorithm for web services discovery optimization with high accuracy and response time is BAT algorithm and CUCKOO search algorithm. CS and BAT are also proven to be much simpler algorithms for optimization search and has significant advantages compared to other metaheuristics algorithm such as ANT, ABC and FF. These advantages are automatic sub grouping which then lead to the ability of dealing with multimodal problems. Another advantage is that the Cuckoo search can produce excellent convergence and high quality solution. FF and $\mathrm{ABC}$ methods however are more complex and need more computational capacity compared to other metaheuristics algorithm.

\section{REFERENCES}

[1]A.Amirthasaravanan, Paul Rodrigues, R. Sudhesh, "Machine driven and effectual web service discovery by hybrid algorithm" International Conference on Mathematics and its Applications 2014 (ICMAA 2014), India, Applied Mathematical Sciences, ,2015,Vol. 9no, pp.3213-3225.

[2]Chaiyakul S., Limapichat K., Dixit A., and Nantajeewarawat E, "A Framework for Semantic Web Service Discovery and Planning", in IEEE Conference on Cybernetics and Intelligent Systems, 2006.

[3]M. Dorigo, T. Stutzle, “Ant Colony optimization”,A Bradford book, MIT Press Cambridge,Massachucettslondon, England,2004.

[4]A. H. Gandomi, Xin-She Yang,"Bat Algorithm: A Novel Approach for Global Engineering Optimization”, Engineering Computations,2012, Vol. 29, Issue 5, pp.464-483. 
[5]IBM Corporation,"Web Service Level Agreement (WSLA) Language Specification" Ver. 1.0. Retrieved from http://www.research.ibm.com/wsla/WSLASpecV120030128.pdf,2006.

[6]D. Karaboga, B. Basturk, "A powerful and efficient algorithm for numerical function optimization: artificial bee colony (ABC) algorithm", Journal of Global Optimization, 2007, pp.459-471.

[7]Kennedy, J.; Eberhart, R. "Particle Swarm Optimization", Proceedings of IEEE International Conference on Neural Networks ,1995, pp. 1942-1948.

[8]Nebil Ben Mabrouk, "QoS-aware Service-Oriented Middleware for Pervasive Environments", $\mathrm{PhD}$ thesis, UNIVERSITÉ PARIS 6, Écoledoctoraleinformatique, télécommunications, électronique,2012.

[9]T. Rajendran, P. Balasubramanie, "An Optimal Agent-Based Architecture for Dynamic Web Service Discovery with QoS", Second International conference on Computing, Communication and Networking Technologies, IEEEXplore,2010.

[10]Ran, S., "A Model for Web Services Discovery with QoS”, SIGEcom Exchanges,2004, Vol. 4(1), pp.1-10.

[11] A. Rekaby, "Directed Artificial Bat Algorithm (DABA)", IEEE International Conference on Advances in Computing, Communications and Informatics (ICACCI),2013.

[12]S. Walton, O. Hassan, K. Morgan, M. R. Brown, “A Review of Development and Application of the Cuckoo Search Algorithm", Swarm Intelligence and Bio-Inspired Computation, 2013, pp.257-271.

[13]W. Wang, Bin Wu, Y. Zhao and D. Feng," Particle Swarm Optimization for Open Vehicle Routing Problem”, ICIC 2006, LNAI 4114, Springer-Verlag, 2006, pp. 999 - 1007. [14]WengeRong, Kecheng Liu, "A Survey of Context Aware Web Service Discovery: From User's Perspective”,Fifth IEEE International Symposium on Service Oriented System Engineering,2010.

[15]Xin-She Yang, "Fire fly algorithm for multimodal optimization", in proceedings of the stochastic Algorithms. Foundations and Applications (SAGA 109 of Lecture notes in Computer Sciences Springer), 2009, vol.5792.

[16]Xin-She Yang, S. Deb, “Cuckoo search via L'evy flights”, in: Proc. of World Congress on Nature \& Biologically Inspired Computing (NaBIC2009), December2009, India. IEEE Publications, USA, 2009, pp. 210-214.

[17]Xin-She Yang,"A New Metaheuristic Bat-Inspired Algorithm”, Nature Inspired Cooperative Strategies for Optimization (NISCO), Springer- Verlag, 2009.

[18]Xin-She Yang, "Firefly algorithm, stochastic test functions and design optimization", International Journal of Bio-Inspired computation, 2010, vol. 2, no. 2, pp. 78-84.

[19]Xin-She Yang,"A new Metaheuristic bat- Inspired Algorithm”, Studies in computational Intelligence, Springer Berlin, 284, Springer,2010,pp. 65- 74. 